\title{
Wavelet Based Intentional Blurring Variance Scheme for Blur Detection in Barcode Images
}

\author{
Shamik Tiwari ${ }^{1}$, V. P. Shukla ${ }^{2}$, S.R. Biradar ${ }^{3}$, Ajay Kr. Singh ${ }^{4}$ \\ Faculty of Engineering \& Technology Mody Institute of Technology \& Science \\ 1shamiktiwari@ hotmail.com, ${ }^{2}$ drsvprasad2k@yahoo.com \\ ${ }^{3}$ srbiradar.et@mitsuniversity.ac.in, ${ }^{4}$ aksingh.et@ mitsuniversity.ac.in
}

\begin{abstract}
Blur is an undesirable phenomenon which appears as one of the most frequent causes of image degradation. Automatic blur detection is extremely enviable to restore barcode image or simply utilize them. That is to assess whether a given image is blurred or not. To detect blur, many algorithms have been proposed. These algorithms are different in their performance, time complexity, precision, and robustness in noisy environments. In this paper, we present an efficient method blur detection in barcode images, with no reference perceptual blur metric using wavelets.
\end{abstract}

Index Terms - Blur, Intentional blur detection, Wavelet, Barcode images.

\section{INTRODUCTION}

These days' barcodes are being widely used in trade market [1, 2]. Almost every consumer product has a unique 1-D or 2-D barcode for identification. Barcodes encode a series of characters or symbols to hold explicit information and a database key. With the use of a barcode laser scanner, product information such as manufacturing details and price can be easily accessed. Consumer can obtain information about a product at home or in a supermarket, with a scanning device that can decode the product's bar code, and a communication device that retrieves the information from a consumer product server. The ease of use of mobile phones with camera facility gives a portable platform for decoding barcode rather than the use of the traditional laser scanner which has lack of portability. Camera phones can take an image of the bar code and after that it can communicate to a consumer product server to access information related with the product. However, using a camera phone in such applications is challenging due to factors such as geometric distortion, noise, and blurring. Image blurring $[3,4]$ is frequently an issue that affects the performance of a barcode identification system. There are two main kinds of blurring: one is motion blur, which is caused by the relative motion between the camera and object during image capture; the other is defocus blur, which is due to the inaccurate focal length adjustment at the time of image capturing. Blurring induces the degradation of image quality, specifically for barcode images where the encoded information is easily lost due to blur.
An image may be blurred due to diverse sources like atmospheric turbulence, defocused lens, optical abnormality, and spatial and temporal sensor assimilation. Human visual system has good capability to perceive it. However, the mechanism behind this capability is not completely understood for application in artificial visual systems. Therefore, it is hard to design a metric for blur estimation in images. Mean Square Error (MSE), PeakSignal-to-Noise Ratio (PSNR) and Structural Similarity Index (SSIM) are few examples of metrics for image quality , but they are by definition based on references, which means that the system needs to have an idea of what an un-blurred image is. We therefore require a mechanism that can detect blur with no use of references. Tong et al. [5] proposed a scheme to decide whether an image is blurred or not and to what extent an image is blurred. They used capability of Harr wavelet transform to discriminate different types of edges and estimate the effect of blur on the edges. Yang et al. [6] addressed the motion blur detection scheme using support vector machine to classify the digital image as blurred or sharp categories. Aizenberg et al. [7] presented a work that considers the blur detection as a pattern classification problem. This work identifies type of blur and estimates blur parameters using neural network for four categories of blur namely defocus, rectangular, motion and Gaussian. Crete et al. [8] used intentional blurring pixel difference (IBD) algorithm due to the fact that it doesn't require the use of edge detection. Another factor in considering IBD algorithm is its computational speed. As the name states, this algorithm is based on intentional blurring of the given image. The principle stated in [8] is straightforward with the observation that, the intentional blurring of a sharp image gives enormous gray scale variations. On the other hand, intentional blurring of an already blurred image gives small gray scale variations. Although it gives details on calculating the blur estimate, the blur decision threshold is not mentioned. The drawback of the suggested method by the authors is that this method fails to identify blur parameters when the blur size is small.

In this paper, we proposed no-reference blur detection in wavelet domain for barcode images blurred with motion blur, defocus blur and co-existence of both blurs. Automatically determining blurred images helps to apply deconvolution method prior to any further image 
processing operation. This paper is organized in five sections including the present section. In section 2, we discuss the theory of image restoration and blur models. Section 3 presents barcode segmentation technique. Section 4 gives overview of discrete wavelet transform. Section 5 is devoted to proposed blur detection scheme and section 6 presents experimental results. In the final section 7 , conclusion is discussed.

\section{IMAGE DEGRADATION MODEL}

The image degradation process can be modelled by the following convolution process as described in $[3,4]$

$$
g(x, y)=f(x, y) * h(x, y)+\eta(x, y)
$$

Where, $g(x, y)$ is the degraded image in spatial domain, $f(x, y)$ is the uncorrupted original image in the spatial domain, $h(x, y)$ is the point spread function that caused the degradation and $\eta(x, y)$ is the additive noise. Since, convolution in spatial domain is equal to the multiplication in frequency domain, equation (1) can be written as:

$$
G(u, v)=F(u, v) H(u, v)+N(u, v)
$$

When the scene to be recorded translates relative to the camera at a constant velocity ( $\mathrm{v}_{\text {relative }}$ ) under an angle of $\alpha$ radians with the horizontal axis during the exposure interval $\left[0, t_{\text {exposure }}\right]$, the distortion is one dimensional. Defining the length of motion as $L=v_{\text {relative }} \times$ $t$,exposure, the PSF in spatial domain can be described as $[9,10]$ :

$$
h(x, y)=\left\{\begin{array}{c}
\frac{1}{L} \text { if } \sqrt{x^{2}+y^{2}} \leq \frac{L}{2} \text { and } \frac{x}{y}=-\tan \alpha \\
0 \quad \text { otherwise }
\end{array}\right.
$$

In most cases, the out of focus blur caused by a system with circular aperture can be modeled as a uniform disk with radius $\mathrm{R}$ as $[11,12]$ :

$$
h(x, y)=\left\{\begin{array}{l}
\frac{1}{\pi R^{2}} \\
0
\end{array}\right.
$$$$
\text { if } \sqrt{x^{2}+y^{2}} \leq R
$$

otherwise

In the case in which both out-of-focus blur and motion blur are present in the same image, the blur model is:

$$
h(x, y)=a(x, y) * b(x, y)
$$

Where $a(x, y), b(x, y)$ are point spread functions for motion and defocus blur respectively and * is convolution operator.

\section{BARCODE SEGMENTATION}

The first step is localization of barcode area in camera based document image. To crop barcode from image we must find two points corresponding to the top-left and bottom-right corner of the barcode area. There exist various approaches for 1-D or 2-D bar code localization using analysis in the spatial domain [13-17], Gabor filtering [18] and analysis in the frequency domain [1920]. We used difference of gradient method [23] for barcode segmentation. The principle behind the approach is that a 1-D barcode consists of vertical bars. Therefore, we may trace the barcode by finding area with high horizontal gradients and low vertical gradients. Based on this idea, we apply following gradient difference operation to an image $f(x, y)$ :

$$
\left|G_{x}\right|-\left|G_{y}\right|
$$

Where $\mid G_{x}$ and $\left|G_{y}\right|$ are absolute values of gradient in $\mathrm{x}$ and $\mathrm{y}$ direction. To calculate gradient we used Sobel operator because it has larger convolution mask that smoothes the input image to a greater extent and so makes the operator less sensitive to noise. The Sobel operator [21] computes the gradient by using the discrete differences between the rows and columns of $3 \times 3$ neighbourhoods using convolution mask as shown in Fig.1.

\begin{tabular}{|l|l|l|}
\hline-1 & 0 & +1 \\
\hline-2 & 0 & +2 \\
\hline-1 & 0 & +1 \\
\hline
\end{tabular}

$\left|G_{x}\right|$

\begin{tabular}{|c|c|c|}
\hline+1 & +2 & +1 \\
\hline 0 & 0 & 0 \\
\hline-1 & -2 & -1 \\
\hline
\end{tabular}

Fig 1: Sobel convolution masks
So the gradient is computed by the following equations where Z's are the intensities arranged as in fig. 2 .

$$
\begin{aligned}
& G x=(Z 7+2 Z 8+Z 9)-(Z 1+2 Z 2+Z 3) \\
& G y=(Z 3+2 Z 6+Z 9)-(Z 1+2 Z 4+Z 7)
\end{aligned}
$$

\begin{tabular}{|l|l|l|}
\hline Z1 & Z2 & Z3 \\
\hline Z4 & Z5 & Z6 \\
\hline Z7 & Z8 & Z9 \\
\hline
\end{tabular}

Fig.2: Image neighbourhood

Afterwards, we low-pass filtered the result to eliminate isolated edges and noise. The point where this operation is maximized is assumed to be located within the barcode From this point, we move outwards to establish precise left and right bounds for the entire barcode. Fig. 3 and 4 shows an image taken by camera and segmented barcode image respectively. This approach gives successful result upto blur extent of 15 pixels. 


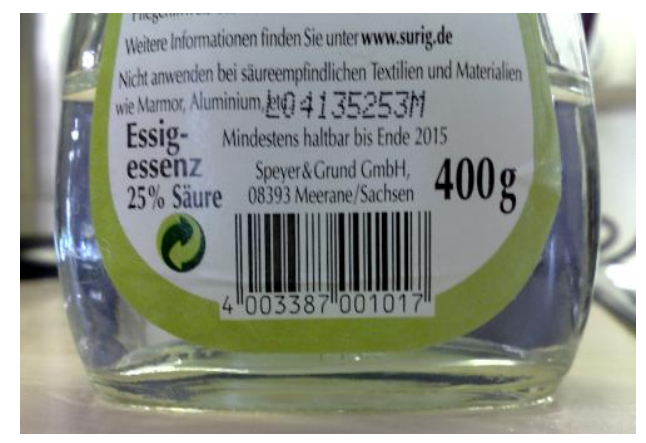

Fig 3: camera image containing barcode

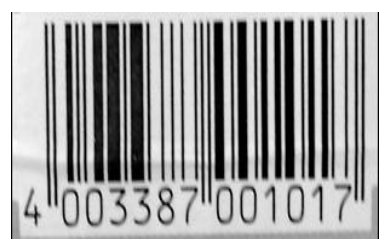

Fig 4: Segmented barcode image

\section{DISCRETE WAVELET TRANSFORM}

The discrete wavelet transform [3, 4] is a dominant and impressionable framework for image processing and analysis tasks. It is broadly used in the fields of image compression, segmentation, de-noising, recognition and fusion etc. The two-dimensional discrete wavelet transform (DWT) hierarchically decompose an input image into a sequence of successively lower resolution images and their associated approximate and detail coefficient images. DWT is implemented by a set of filters, which are convolved with the image rows and columns. An image is convolved with low-pass and highpass filters and the odd samples of the filtered outputs are discarded resulting in down sampling the image by a factor of 2 . The $l$ level wavelet decomposition of an image $I$ results in an approximation image $X_{l}$ and three detail images $H, V_{l}$, and $D_{l}$ in horizontal, vertical, and diagonal directions respectively. A barcode image contains a pattern of parallel adjacent bars and spaces, which are aligned vertically. Therefore the barcode area should be evidently dominated with vertical textures. So, in the different subbands of the wavelet image, the coefficients of $H L$ subbands from the barcode area are greater than the $L H$ subbands or $H H$ subbands.

\section{BLUR DETECTION SCHEME}

Our algorithm is based on the phenomena of intentional blurring pixel difference (IBD) algorithm proposed by crest et al. [8]. This blur detection scheme works on the principle that it is difficult to observe difference between blurred image and re-blurred version of same image. It can be easily observed that when we blur sharp image there is high loss of details but reblurring of blurred image causes low loss. Fig.5 demonstrates the concept.

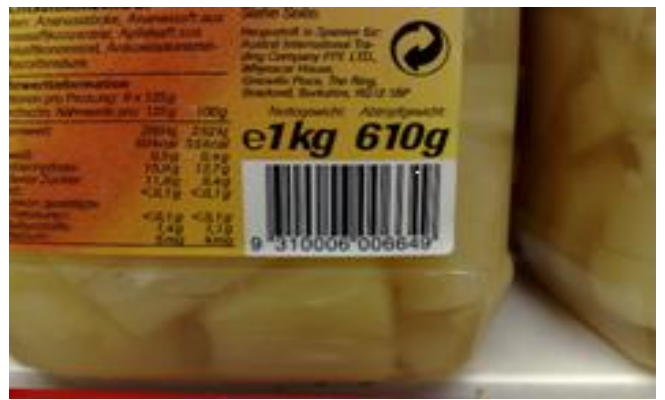

(a)

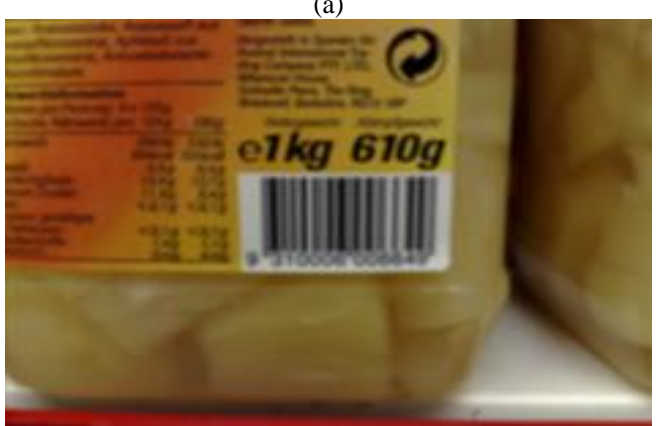

(b)

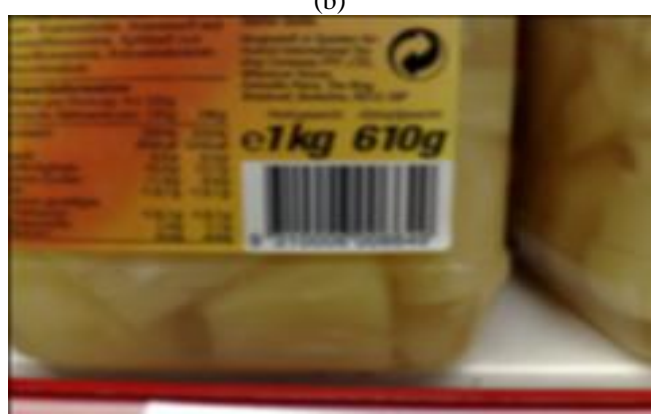

(c)

Fig 5: (a) Original sharp image, (b) blurred version of (a), (c) reblurred version of (b) with same extent 


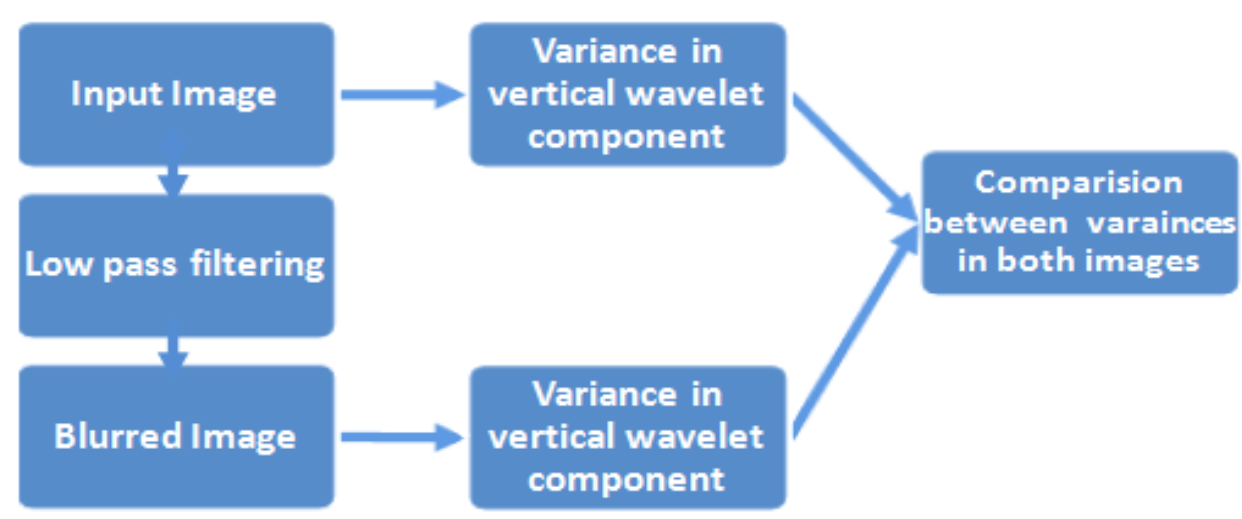

Fig 6: Block diagram of intentional blur algorithm

With the inspiration of intentional blurring scheme, we are able to detect blur on an image by blurring it and comparing the variations in vertical wavelet coefficients before and after the low-pass filtering step. As a result, the first step consists in the estimation of the intensity variations in $\mathrm{V}_{1}$ of the input image. On this same image, we apply a low-pass filter and compute variations in $\mathrm{V}_{1}$ components. Then, the comparison between these variations allows us to evaluate the blur influence. Thus, a high variation between the original and the blurred image means that the original image was sharp whereas a slight variation between the original and the blurred image means that the original image was already blurred. This description is summarized in Fig.6.

The blur detection algorithm starts by filtering the original image with low pass filter in spatial domain as given in equation (10).

$$
h=\frac{1}{9}\left[\begin{array}{lll}
1 & 1 & 1 \\
1 & 1 & 1 \\
1 & 1 & 1
\end{array}\right]
$$

The filtered image is obtained by convolution of input image $f(x, y)$ and filter function $h$.

$$
b(x, y)=h * f(x, y)
$$

To analyze variance in vertical details of barcode region compute the Haar wavelet transform of input image and blurred image both using equation :

$$
T=H F H^{T}
$$

Where $\mathrm{F}$ is an $\mathrm{NXN}$ image matrix, $\mathrm{H}$ is a $\mathrm{N} \mathrm{XN}$ Haar transformation matrix, and $\mathrm{T}$ is the resulting $\mathrm{NXN}$ transform. For the Haar transform, $\mathrm{H}$ contains the Haar basis functions, $h_{k}(z)$. They are defined over continuous interval $\mathrm{z} \in[0,1]$ for $\mathrm{k}=0,1,2, \ldots \ldots \ldots \mathrm{N}-1$, where $\mathrm{N}=2^{\mathrm{n}}$. To generate $\mathrm{H}$, define the integer $\mathrm{k}$ such that $\mathrm{k}=2^{\mathrm{p}}+\mathrm{q}-1$, where $0 \leq \mathrm{p} \leq \mathrm{n}-1, \mathrm{q}=0$ or 1 for $\mathrm{p}=0$, and $1 \leq \mathrm{q} \leq 2^{\mathrm{p}}$ for $\mathrm{p} \neq 0$. Then the Haar basis functions are:

$$
h_{0}=h_{00}(z)=\frac{1}{\sqrt{N}}, \quad z \in[0,1]
$$

And

$$
\begin{aligned}
h_{k}(z) & =h_{p q}(z) \\
& =\frac{1}{\sqrt{N}}\left\{\begin{array}{cr}
2^{\frac{p}{2}} & \frac{q-1}{2^{p}} \leq z<\frac{q-0.5}{2^{p}} \\
-2^{\frac{p}{2}} & \frac{q-0.5}{2^{p}} \leq z<\frac{q}{2^{p}} \\
0 & \text { otherwise }, z \in[0,1]
\end{array}\right.
\end{aligned}
$$

After computing Haar transformation, compute the variance in vertical detail coefficient of input image $\left(V_{f}\right)$ and blurred image $\left(V_{b}\right)$ in vertical direction as given below.

$$
\begin{gathered}
V a r_{-} V_{f}=\frac{1}{M} \sum_{x=0}^{M-1}\left(V_{f}(x, y)-\overline{V_{f}(x, y)}\right) \\
V a r_{-} V_{b}=\frac{1}{M} \sum_{x=0}^{M-1}\left(V_{b}(x, y)-\overline{V_{b}(x, y)}\right)
\end{gathered}
$$

where,

$$
\begin{gathered}
\overline{V_{f}(x, y)}=\frac{1}{M} \sum_{x=0}^{M-1} V_{f}(x, y) \\
\overline{V_{b}(x, y)}=\frac{1}{M} \sum_{x=0}^{M-1} V_{b}(x, y)
\end{gathered}
$$

for $x=0,1, \ldots, M-1$, and $y=0,1, \ldots \ldots, N-1$.

In order to analyze the variance of vertical detail coefficients after blurring and compare with the variance of vertical detail coefficients before blurring, variance absolute difference is computed by

$$
A_{-} \text {Var }=\max \left(0, V a r_{-} V_{f}-V a r_{-} V_{b}\right)
$$

Then find the sum of coefficients in $V a r_{-} V_{f}$ and A_Var as:

$$
\begin{aligned}
& S_{-} V a r_{-} V_{f}=\sum_{y=0}^{N-1} \operatorname{Var}_{V_{f}} \\
& S_{-} A \_V a r=\sum_{y=0}^{N-1} A \_V a r
\end{aligned}
$$

To get final blur factor normalize above sum 
coefficients using

$$
B F=\left(\frac{S_{\operatorname{Var}_{V_{f}}}-S_{A_{V a r}}}{S_{V a r_{V_{f}}}}\right)
$$

It is evident that the higher the value of the blur factor $(\mathrm{BF})$, it is more accepted that the input image was blurred.

\section{SIMULATION RESULTS}

The performance of the proposed technique has been evaluated using many camera based barcode images. The WWU Muenster Barcode Database [22] of barcode images has been used in order to carry out experimental work. We have taken 100 barcode images. Then, the three different classes of blur (motion, defocus and mixed) were synthetically introduced with different parameters to make the databases of 4000 images (i.e., 1000 images with each class of blur and 1000 without blur). All the algorithms were implemented in MATLAB running on Intel Core2Duo machine with $2.40 \mathrm{GHz}$ processor and 2 GB of RAM memory. The threshold value chosen for blur detection is .4 for both IBD scheme and proposed method.

Table 1: Results

\begin{tabular}{|l|c|c|}
\hline \multirow{2}{*}{ Blur Type } & \multicolumn{2}{|c|}{ Accuracy (\%) } \\
\cline { 2 - 3 } & IBD method [8] & $\begin{array}{c}\text { Proposed } \\
\text { method }\end{array}$ \\
\hline Motion & 90.4 & 96.8 \\
\hline Defocus & 99.2 & 100 \\
\hline Combined & 96.2 & 100 \\
\hline No-Blur & 74.4 & 98.8 \\
\hline
\end{tabular}

Images are contaminated by different types of noise. Most common types of noise are impulsive and Gaussian noise, which affect the image at the time of acquisition due to noisy sensors. Noise also contaminates the image during transmission due to channel errors. Although there are different noise models, this work confines to dealing with blur in the occurrence of Gaussian noise which is the most common scenario in practical applications [3, 4]. The Gaussian noise model is expressed as:

$$
\eta(x, y)=\frac{1}{2 \pi \sigma^{2}} e^{-\left(x^{2}+y^{2} / 2 \sigma^{2}\right)}
$$

It is characterized by its variance term $\sigma^{2}$.

In order to examine the robustness of the proposed method in presence of noise, additive Gaussian noise of $20 \mathrm{db}$ SNR is added to create noisy blurred images. Table 2 illustrates the results in presence of noise.
Table2: Results in presence of $20 \mathrm{db}$ noise

\begin{tabular}{|l|c|c|}
\hline \multirow{2}{*}{ Blur Type } & \multicolumn{2}{|c|}{ Accuracy (\%) } \\
\cline { 2 - 3 } & IBD method [8] & $\begin{array}{c}\text { Proposed } \\
\text { method }\end{array}$ \\
\hline Motion & 82.2 & 92.8 \\
\hline Defocus & 92.2 & 94.5 \\
\hline Combined & 88.4 & 95.3 \\
\hline No-Blur & 54.4 & 94.2 \\
\hline
\end{tabular}

The simulation results are listed in table 1 and table 2 . Results demonstrate the effectiveness of our scheme over proposed by authors in [8] for determining blur. Fig.7 shows few results for blur detection in case of motion blurred, defocus blurred and combined blur in barcode images.

\section{CONCLUSION}

In this paper, we have proposed a new blur detection scheme for barcode images taken by digital cameras. The scheme is based on intentional blur variance scheme of wavelet coefficients to discriminate blurred or sharp images. Experimental results show the higher blur detection accuracy compared to the other method without noise as well as in presence of noise. In the proposed algorithm, to achieve good result, threshold has been experimentally set. Automatically determining threshold value needs to further researches.

\section{ACKNOWLEDGEMENT}

We highly appreciate Faculty of Engineering and Technology, Mody Institute of Technology \& Science University, Laxmangarh for providing facility to carry out this research work.

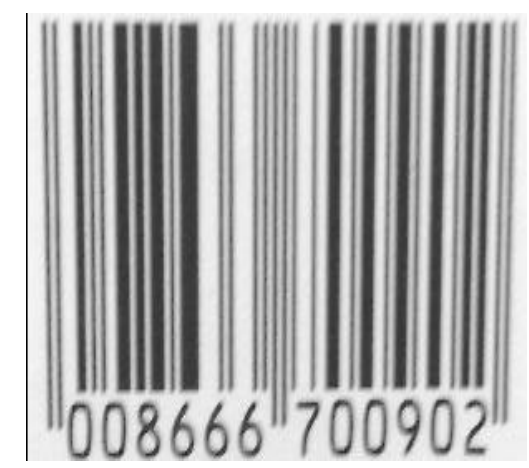

(a) Original Image (No Blur) Blur Factor .0062 


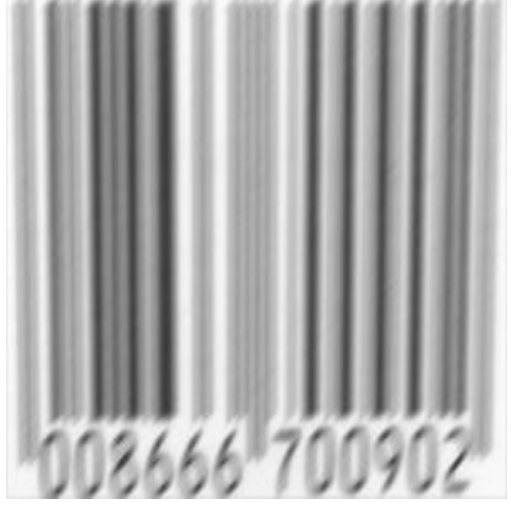

(b) Motion Blurred Image Blur Factor 9912

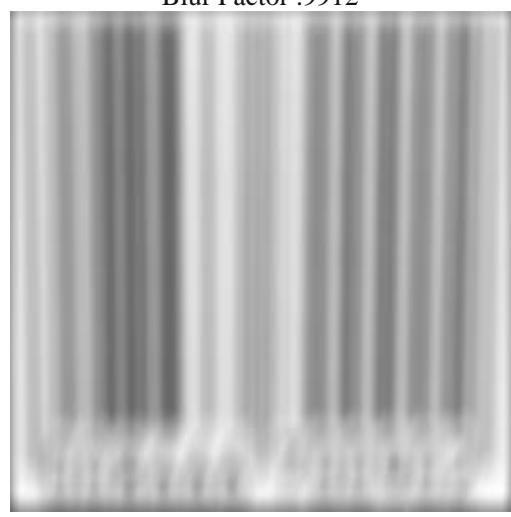

(c) Defocus Blurred Image

Blur Factor .9904

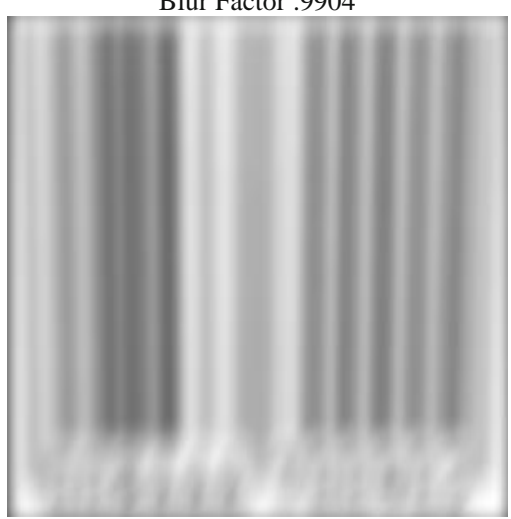

(d) Combined Blurred Image

Blur Factor 9958

Fig 7: An example of the proposed scheme to determine the blur factor in different blur categories

\section{REFERENCES}

[1] J. Vartiainen, T. Kallonen, and J. Ikonen, "Barcodes and Mobile Phones as Part of Logistic Chain in Construction Industry," The 16th International Conference on Software, Telecommunication, and Computer Networks, pp. $305-$ 308, September 25-27, 2008.

[2] K. Q. Wang, "Barcode character segmentation for mobile cameras", technical report of NOKIA, 2003.

[3] Jain A.K., "Fundamentals of Digital Image Processing", Prentice Hall International, 1989.

[4] Gonzalez R.C., Woods R.E., "Digital Image Processing”, Prentice Hall, 2007.
[5] Tong H., Li M., Zhang H., Zhang C., "Blur detection for digital images using wavelet transform," in proceedings of IEEE international conference on Multimedia and Expo, Vol. 1, pp. 17-20, 2004.

[6] Kai-Chieh Yang, Clark C. Guest and Pankaj Das, "Motion blur detecting by support vector machine", Proc. SPIE 5916, 59160R, 2005.

[7] I. Aizenberg, N. Aizenberg, T. Bregin, C. Butakov, E. Farberov, N. Merzlyakov, O. Milukova "Blur Recognition on the Neural Network based on Multi-Valued Neurons", Journal of Image and Graphics. Vol.5, 2000.

[8] Crete F., Dolmiere T., Ladret P., Nicolas M., "The Blur Effect: Perception and Estimation with a New NoReference Perceptual Blur Metric," SPIE Human Vision \& Electronic Imaging, Vol.6492, 64920I, pp. 64920I-164920I-011, 2007.

[9] M. Cannon, "Blind Deconvolution of Spatially Invariant Image Blurs with Phase", IEEE Trans. Acoust. Speech Signal Processing, Vol. 24(1), pp. 56-63,1976.

[10] F. Krahmer, Y. Lin, B. McAdoo, K. Ott, J. Wang, D. Widemann, B. Wohlberg, "Blind Image Deconvolution: Motion Blur Estimation", Tech Rep., Univ. Minnesota, 2006.

[11] Moghaddam M.E., "A Mathematical Model to Estimate Out of Focus Blur," in proceedings of 5th IEEE international symposium on Image and Signal Processing and Analysis, pp. 278-281, 2007.

[12] Sakano M., Suetake N., Uchino E., "A robust Point Spread Function estimation for Out-of-Focus Blurred and Noisy Images based on a distribution of Gradient vectors on the polar plane", Journal of Optical Society of Japan, co-published with Springer-Verlag GmbH, Vol. 14, No. 5, pp. 297-303, 2007.

[13] D. Chai and F. Hock, "Locating and decoding EAN-13 barcodes from images captured by digital cameras", in Proc. IEEE ICICS'05, pp. 1556-1560, Dec. 2005.

[14] R. Howlett, S. Berthier, and G. Awcock, "Determining the location of industrial bar-codes using neural networks", in Proc. IEE IPA'97, vol. 2, pp. 511-515, July 1997.

[15] S.J. Liu, H.Y. Liao, L.H. Chen, H.R. Tyan, and J. W. Hsieh, "Camera-based bar code recognition system using neural net”, in Proc. IEEE IJCNN'93, vol. 2, Nagoya, pp. 1301-1305, Oct. 1993.

[16] S. Arnould, G. Awcock, R. Thomas, "Remote barcode localisation using mathematical morphology", in Proc. IEEE IPA'93, vol. 2, pp. 642-646, July 1999.

[17] C. Viard Gaudin, N. Normand, D. Barba, "A bar code location algorithm using a two-dimensional approach", in Proc. IEEE ICDAR'93, pp. 45-48, Oct. 1993.

[18] A. K. Jain and Y. Chen, "Bar code localization using texture analysis," in Proc. IEEE ICDAR'93,pp. 41-44, Oct. 1993.

[19] Tropf Alexander, Douglas Chai.,"Locating 1-D Bar Codes in DCT-Domain", ICASSP2, pp. 741-44, 2006.

[20] R. Oktem, "Bar code localization in wavelet domain by using binary morphology," in Proc. IEEE SIU'04, pp. 499-501, Apr. 2004.

[21] Wenshuo Gao, Lei Yang, Xiaoguang Zhang, Huizhong Liu, "An Improved Sobel Edge Detection", 3rd IEEE International Conference on Computer Science and Information Technology (ICCSIT), Vol-5, pp.67 - 71, 2010.

[22] S. Wachenfeld, S. Terlunen, X. Jiang: http://cvpr.unimuenster.de/ research/barcode.

[23] Shamik Tiwari, V. P. Shukla, S. R. Biradar, Ajay Kumar Singh, "Texture Features based Blur Classification in 
Barcode Images", I.J. Information Engineering and Electronic Business, MECS Publisher, vol. 5, pp. 34-41, 2013.

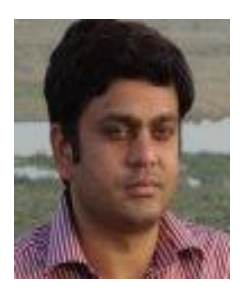

Shamik Tiwari was born in India, in 1981. $\mathrm{He}$ received his B.E. (Computer Sc. \& Engineering) in 2003, M.Tech. (Computer Sc. \& Engg.) in 2007 from RGPV University Bhopal and Dr. B. R. Ambedkar University Agra respectively. He has joined as an Asst. Professor in Mody Institute of Technology \& Science, Deemed University Laxmangarh in 2009. Presently, he is pursuing Ph.D. in Computer Sc. \& Engg. from the MITS Lakshmangarh. He has published over 15 papers in refereed journals and conference proceedings. He is an author of the book "Digital Image Processing" from Dhanpat Rai Publishing (India), His current research interest includes digital image processing, Pattern Classification, and their applications in computer vision.

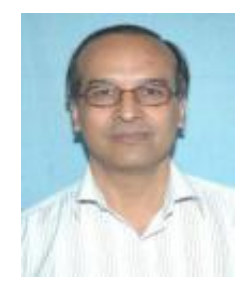

Vidya Prasad Shukla was born in India, in 1954. He received his M.Sc. (Applied Mathematics) in 1976, Ph.D. (Modelling and Computer Simulation) in 1982 and PG Dip. (Computational Hydraulic Engineering) in 1986 from Avadh University Faizabad, Indian Institute of Technology Kanpur and International Institute of Environmental \& Hudraulic Engineering (Delft) the Netherlands respectively. He worked and officiated at various posts as Senior Research Officer, Chief Research Officer and HOD Computer Division at from Central Water and Power research Station (CWPRS), Pune from 1982 to 2003. Thereafter, he worked as a Professor in BIT, Sathyamangalam and NIT Durgapur. He has joined as a Professor in Mody Institute of Technology \& Science, Deemed University Laxmangarh in 2009. He has published over 57 papers in refereed journals and conference proceedings and written 29 technical reports on various clients sponsored research projects of international/national importance. He is an editor of the book "Development of Coastal Engineering" from CWPRS, Pune. His current research interest includes Computer Simulation \& Modeling, Image processing, Cellular Automata, SoftComputing, Computer Vision, Nanotech-simulation, Operations Research, Mathematical Biology, Modeling of Arms Race of Nations.

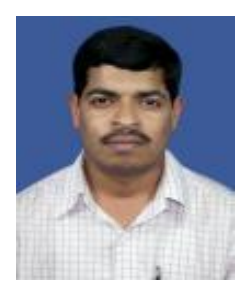

S.R. Biradar is a Professor in the department of Computer Science and Engineering, MITS, Lakshmangarh, India. $\mathrm{He}$ received his B.E, M.Tech and Ph.D degrees in Computer Science and Engineering from Karnataka University, MAHE Manipal and Jadavpur University respectively. His research interest includes Mobile Ad-hoc networking, advanced wireless communication. $\mathrm{He}$ has joined as Professor in Mody Institute of Technology \& Science, Deemed University Laxmangarh in 2011. He has published over 45 papers in refereed journals and conference proceedings. His current research interest includes Image Processing, Mobile ad hoc networks, and sensor networks.

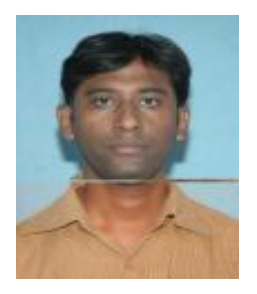

Ajay Kumar Singh was born in India, in 1980. He received his B.E. (Computer Sc. \& Engineering) in 2001, M.Tech. (Information Technology) in 2006 from CCS University Meerut and AAI Deemed University Allahabad respectively. He has joined as an Asst. Prof. in Mody Institute of Technology \& Science, Deemed University Laxmangarh in 2009. Presently, he is pursuing Phd. in Computer Sc. \& Engg. from the MITS Lakshmangarh. He has published over 10 papers in refereed journals and conference proceedings. His current research interest includes Image Processing, Image classification and their applications in computer vision. 